\title{
Virtual Project Teaming: Incorporation of Immersive Environments and Role- played Case Study Assessments
}

\section{Dr. Charles J Lesko Jr., East Carolina University}

Dr. Charles J. Lesko, Jr. is currently serving as Assistant Professor in the College of Technology \& Computer Science at East Carolina University instructing at both the graduate and undergraduate-level course levels for the Department of Technology Systems. His current teaching and research regime focus on Information Technology Project Management, and Virtually Immersive Technologies. Throughout the course of his career, Dr. Lesko's focus has been on managing and leading technological innovation in the workplace; his past experiences bring to the table a heavy technical background with a strong management and leadership base. He has over (18) years of experience in systems integration and project management fields. His experience base prior also includes (12) year's service as an officer in the United States Marine Corps. He can be reached 252-737-1907 or at leskoc@ecu.edu. 


\title{
Virtual Project Teaming: Incorporation of Immersive Environments and Role-played Case Study Assessments
}

\begin{abstract}
The purpose of this article is to present a role-play based approach to virtual project teaming and assessment that provides graduate students with collaborative working spaces, a context within which students can interact with their peers, and a structured approach for facilitators to evaluate the efforts of these virtual teams. Content and analytics for this study stem from data collected over the course of three semesters while offering a graduate-level Technology Project Management course. To offer this online course, several virtual working spaces were utilized to present course lectures and discussions, and to provide virtual in-world team skills training.

Discussion includes the incorporation of virtual role-play centered on the use of a contextually appropriate case study that spans a period 10 of the 14 weeks of the semester-long course. The article also addresses both the structure of the course delivery. The case study and role play structure, and challenges faced in maximizing the immersive capabilities of these types of virtual environments all while ensuring that content delivery and required course assessments were being realized. A summary of student survey data and discussion of lessons learned is also incorporated.
\end{abstract}

\section{Introduction}

The recent growth of online educational delivery has in many ways paralleled the need for virtual collaboration and teaming in the corporate setting. ${ }^{1,2}$ In many respects, delivering an online class has many of the same challenges that corporate virtual teams face. ${ }^{3,4}$ These similarities become even more obvious when the online course topic involves virtual project teaming. ${ }^{5}$ The advantages of virtualized teams have been evidenced throughout both industry and academia. ${ }^{6}$ Recent studies indicate most corporation's measure the performance of their project teams by cost, time, and quality and that they have found that decentralizing project teams "offers opportunities to save on time and costs, and still achieve quality results".

Any reference to today's current virtual reality solutions as being 'immersive' in nature typically connotes use of a digital technology capability that can "deeply involve one's senses". ${ }^{8}$ In his overview on immersive environments, Miles notes that creating and utilizing these immersive spaces is "driven by a desire to incorporate as much richness, detail, depth, and subtlety as possible in order to give an individual experiencing the environment the sensation of being totally immersed".?

Incorporating virtually immersive academic workspaces for virtual student teams, especially for an online project management course, can provide significant opportunities for both students and facilitators. ${ }^{10,11}$ Beyond the virtual technologies however, it is also important for academics to clearly outline the team structure, processes, and roles, in order to reduce ambiguity and align expectations. ${ }^{12,13}$ In order to ensure that these teams are successful, a critical first step is determining the virtual media and tools that the offering will use. The technology planning for 
the course offering must account for everything from basic infrastructure to innovative tools that enhance frequent communications.

Many organizations are also using team collaboration tools, such as online white boarding, shared applications, and team workspaces, enabling dispersed employees to work together more effectively. ${ }^{14,15}$ Furthermore, forward-looking organizations are adopting social media tools, such as blogs, podcasts, and wikis to help teams collaborate and gain a sense of

community. ${ }^{16,17,18}$ A virtual team requires not only the right infrastructure, but also a communications plan that describes the tools team members can use to communicate and sets expectations for when to use each.

This article covers the first phase of a long-term study that has been ongoing since 2009; specifically, the article focuses on the course delivery of a graduate-level online course titled: 'Technology Project Management'. The article addresses both structure of the course delivery and challenges faced in maximizing the immersive capability while realizing required content delivery and assessments. A brief summary of some recent student perception survey data and discussion of lessons learned is also included.

\section{Research Objectives}

The overarching objective of this phase of the research effort has been to gain a better understanding of the practical challenges associated with the development and integration of virtual world technologies into a graduate course that involves extensive virtual teaming. Furthermore, over the course of the study to-date, the need to embrace the concept of virtual immersion through the inclusion of more interactive activity has become more evident.

\section{Methodology}

To better understand the challenges of developing collaborative workspaces in a virtually immersive environment both survey and observational analysis have been incorporated. This research analysis has included:

1. Development and incorporation of various virtual collaborative workspaces to support the course delivery.

2. An online, anonymous 'Initial Second Life Experience Survey' was developed to evaluate the early interactions of the students with the virtual world environment Second Life and specifically the population background, initial learning curve students experienced, avatar interaction, and perceived effectiveness of the virtual world medium. This survey is part of an ongoing five-year research effort that began in 2009.

3. Case study materials were developed that challenged the virtual teams to complete weekly tasking common to technology projects.

4. Organized role play for the ongoing case study was developed to give students the opportunity to view project team activities from the stakeholder's perspective. Role play also looks to improve student interaction within the virtual space, an area that early research indicates is lacking in utilization of early course structure. Research suggests 
that the use of role play with virtual teams adds to clarity of course goals and roles and the presence of engagement and cooperation. ${ }^{19,20}$

5. Finally, structured observation has also been incorporated into this study, where appropriate, to evaluate challenges associated with course delivery and management. ${ }^{21,22}$

\section{Course Objectives Relative to this Study}

The recent focus of this ongoing study centers on the delivery of an online graduate-level 'Technology Project Management' course. The course is designed to place into context and then evaluate student performance as technology-focused project team members in the key knowledge areas of project management including project integration, scope, time, cost, quality, human resource, communications, risk, and procurement management. ${ }^{23,24}$ The course structure provides students with adequate preparation and grounding in the tools and techniques for managing technology projects, especially in a virtual or geographically disbursed environment. An overarching premise to this course centers on the need for technology leaders to ably work and manage in group settings, and to synthesize ongoing project data and present that data effectively.

This specific course is part of a distance education curriculum and as such requires delivery of content and assessment via various online tools across both campus provided internal network resources and the Internet. The course syllabus outlines nine key course objectives; however, specific to this research effort, the following course objectives were considered for the current study efforts - that upon completion of this course, each student will be able to:

1. Apply project management concepts by working on a group project as either project manager or active team member.

2. Demonstrate the use of various project management applications to help plan and manage a technology project.

3. Demonstrate knowledge and use of methodologies used to manage cross-functional teams in a global context.

\section{Structure of the Online Course Delivery}

Students were instructed at the beginning of each semester that this course was being offered via the universities Second Life virtual campus and that a single (90) minute online synchronous class session was conducted each week. The primary modes of communication for the course were Blackboard (the institution-wide online learning management solution), Second Life (a virtual world solution used for both synchronous and asynchronous delivery), and the university supported email. The class met synchronously on a weekly basis throughout the semester for a single $1 \frac{1}{2}$ hour session and students had access to the Second Life course site 24/7 for individual use or for as-needed project team collaborations.

Specific to this research, student assessments utilized a variety of factors including: attendance, participation and role-play; weekly case study assignments; team presentations, and final project out-brief. For the case study, each class was divided into several project teams of (4-6) students per team. Each project team was charged with the responsibility for preparing a (12-15) minute 
presentation each week based on the project case study assignment for that week. The presentation was conducted using Second Life. Project teams were instructed that they may incorporate other online communicative media (i.e. audio clips, video, etc.) but the logistics and control of these media were strictly left up to the team. Individual project team meetings to coordinate team activities were the sole responsibility of the project team members; how many meetings and how often they tool place was all up to the team. Each team had a Second Life virtual working space (referred to as a sky lab) for each project team to use to coordinate and practice their presentations in.

The project teams presented an assigned series of tasks as a part of the case study that was given out each week. Each presentation must include a slide presentation that included introductory, agenda, content specific to each task, and summary slides. The project teams were evaluated utilizing a team presentation rubric and then each student received a grade based on their participation and presentation as a team member with additional consideration given on an individual basis for individual efforts that exceeded expectation. The team presentation rubric evaluated the team collectively in several areas including: 'Team Collaboration', 'Presentation Layout/Organization', 'Speaking/Verbal Skills', 'Presentation Structure/Mechanics', 'Task Solution, and 'Question \& Answer'. Assessment categories included: 'Met Expectation', 'Missed a Key Element', 'Missed Key Elements', and 'Failed to Meet Expectation'.

In an effort to improve on the interactive nature of the team assignments and to take advantage of the immersive capabilities inherent in the virtual world working spaces, role play was incorporated into the case study assignments. Students were assigned a role the first night of class and role play for the case study began usually on the third session (see Table 1). Each Student was given guidance on what was expected out of their role player and each role was set so that each student played their role twice during the course.

\begin{tabular}{|c|c|c|c|c|c|c|c|c|c|c|c|c|}
\hline & & & Week 3 & Week 4 & Week 5 & Week 6 & Week 7 & Week : & Week 9 & Week 10 & Week 11 & Week 12 \\
\hline \multirow[t]{2}{*}{ POSITION } & \multirow{2}{*}{$\begin{array}{l}\text { PRON } \\
\text { TEAM }\end{array}$} & \multirow[t]{2}{*}{ DEPT } & $\begin{array}{c}\text { Project } \\
\text { Overview } \\
\end{array}$ & $\begin{array}{c}\begin{array}{c}\text { Strategy } 8 \\
\text { Selection }\end{array} \\
\end{array}$ & \begin{tabular}{|l|} 
Structure \\
8 Chatter \\
\end{tabular} & $\begin{array}{c}\text { Scope \& } \\
\text { wBS }\end{array}$ & Comm & Time & Cost & HR & $\begin{array}{c}\text { lssues \& } \\
\text { Risk }\end{array}$ & Ethics \\
\hline & & & Assign 1 & Assign 2 & Assign 3 & Assign 4 & Assign 5 & Assign 6 & Assign 7 & Assign 8 & Assign 9 & Assign 10 \\
\hline VP Human Resouices/CHIO & A & Adrinin Dept & $x$ & & & & & & & & $x$ & \\
\hline Adrninistrative Assistant & A & Admin Dept & & & & & $x$ & & & $x$ & & \\
\hline HR Spocialist & A & Admin Dept & & & & $x$ & & $x$ & & & & \\
\hline VP Frimcal Resources/CFO & A & Adtran Dept & & $x$ & & & & & $x$ & & & \\
\hline Financia: Anałyst & A & Admin Dept & & & $\mathrm{x}$ & & & & & & & $x$ \\
\hline Accounting Specialist & A & Admin Dept & & & & & & & & & & \\
\hline President:CEO & B & Corp HQ & & $\mathrm{x}$ & & & & & & & & $\mathrm{x}$ \\
\hline Senox VP of OpsicoO & B & Corp HQ & & & & $x$ & & & $\mathrm{x}$ & & & \\
\hline Adminiszrative Officer & B & Corp HQ & $x$ & & & & & & & $\mathrm{x}$ & & \\
\hline Pisk Management OfficeriCRO & B & Corp $\mathrm{HO}$ & & & & & $\mathrm{x}$ & & & & $x$ & \\
\hline Quallity Assurance $\mathrm{Mgr}$ & $\theta$ & Corp HQ & & & $\mathrm{x}$ & & & $x$ & & & & \\
\hline VP of Markesting CMO & 8 & Corp HQ & & & & & & & & & & \\
\hline VP of info \& Tecticio & c & Tech Dept & & $x$ & & & & & & & $x$ & \\
\hline Systems Administrator & c & Tech Dept & $x$ & & & & & $x$ & & & & \\
\hline Sr. Network Analyst & c & Tech Dept & & & & $x$ & & & & & & \\
\hline St. DBANDat Processing Supr. & c & Tech Dept & & & $x$ & & & & $x$ & & & \\
\hline Cal Cemer Manager & c & Tech Dept & & & & & $x$ & & & & & $\mathrm{x}$ \\
\hline Systems Analyst & c & Tech Dese & & & & & & & & & & \\
\hline
\end{tabular}

Table 1: Role Player Weekly Schedule 
Virtual Classroom: Within the institutions already existent virtual campus setting, several virtual workspaces were either created or existing spaces modified to provide the appropriate virtual work environment for the course activities. The first virtual space is a classroom (see Figure 1). This space provides an initial meeting room for all synchronous sessions. Students logged in here and through an activity bot (a proximity counter program) attendance was automatically collected. The auditorium style seating gave plenty of space for all and provided visibility to five separate boards in the front. Having multiple presentation screens allowed the instructor to present several aspects of the course at once, including: class agenda, case study, reading assignments, video clips, quiz/exam results, and presentation slides.

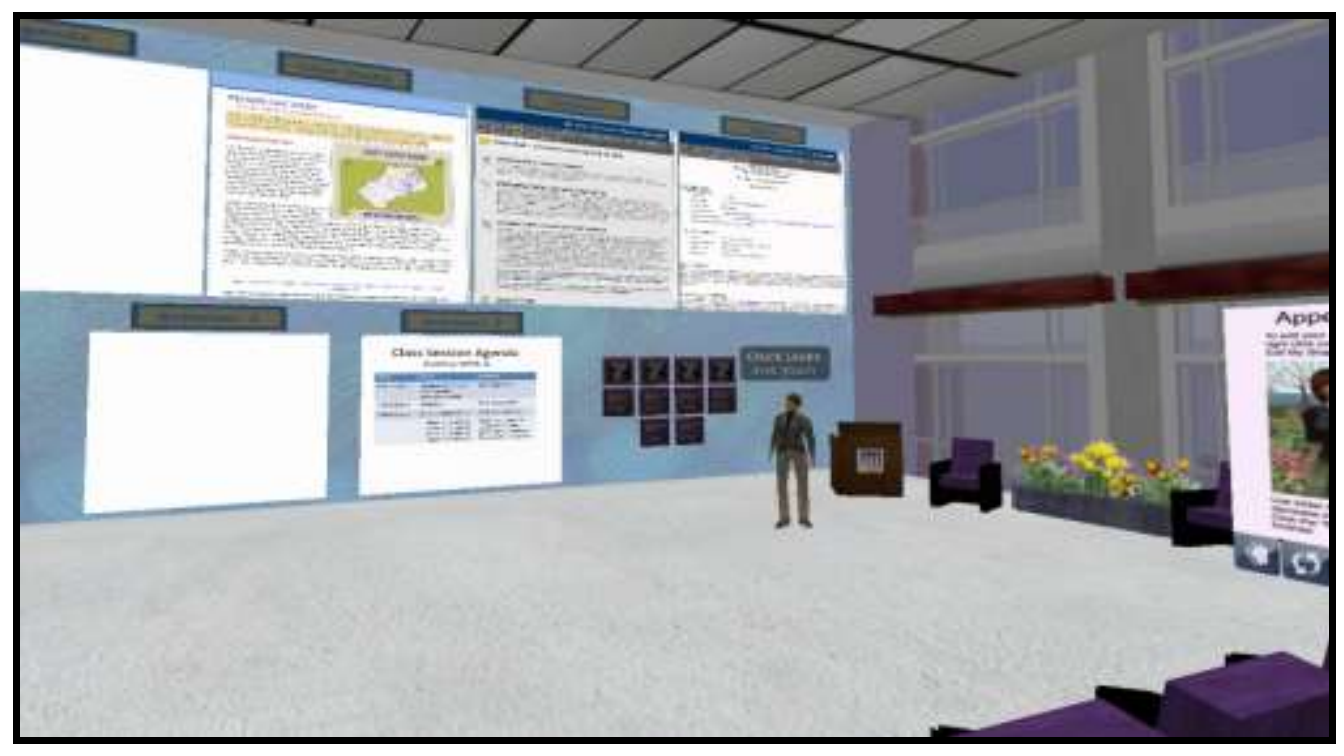

Figure 1: Virtual Classroom/Lecture Workspace

Project Management Interactive Lab: The second virtual space that was utilized was a project management interactive lab building (see Figure 2). This space contains a lobby floor with access to four floors above it. Each of the first three floors above the lobby housed eight learning modules. Each learning module consisted of four viewing stations that was completed by the student completes with the fourth as a review station. The viewing stations presented a series of 12-18 slides, on a timed presentation with each slide presentation lasting approximately 4-6 minutes each. Each course week students were assigned learning modules to complete. Use of this method allowed for presentation of key course content to the students while affording them with the opportunity to view the content at their convenience - asynchronously. 


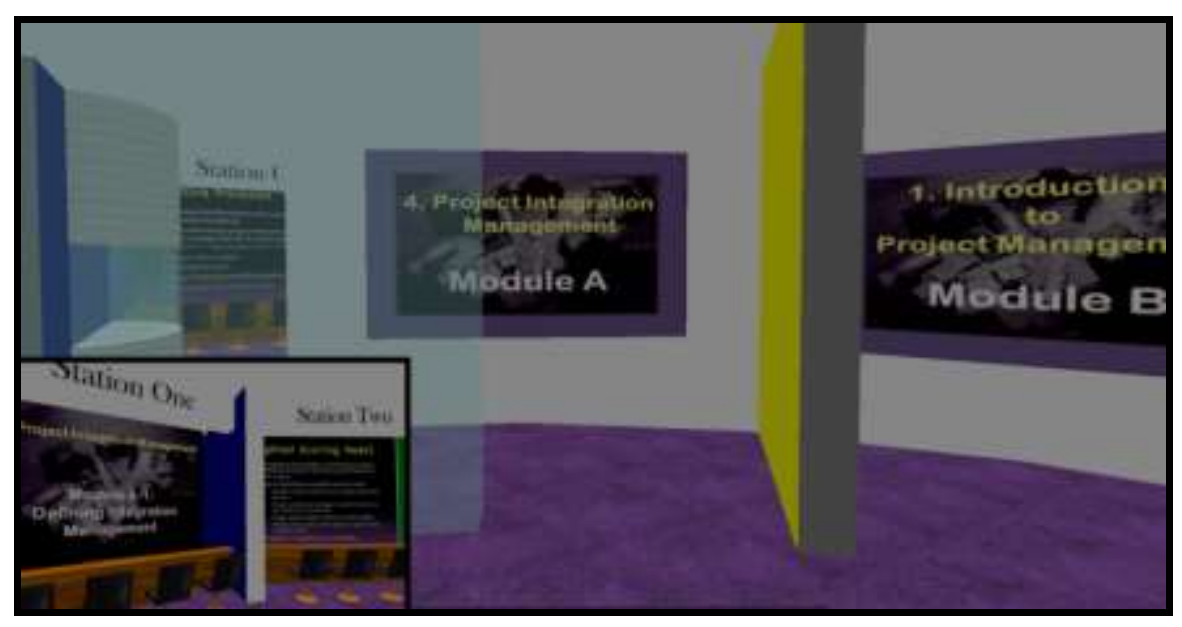

Figure 2: Project Management Interactive Lab

Project Team Sky Labs: The next virtual workspaces (referred to as 'Sky Labs') were created for the student project teams to collaborate in (see Figure 3). These virtual workspaces (floating above the virtual campus) gave each of the virtual project teams a collaborative space to work from. Each of the Sky Labs had enough seating for the (4-6) team members and any visitors. Presentation screens were provided for team members to view case study tasking's and to create team presentations via various online document development and sharing tools.

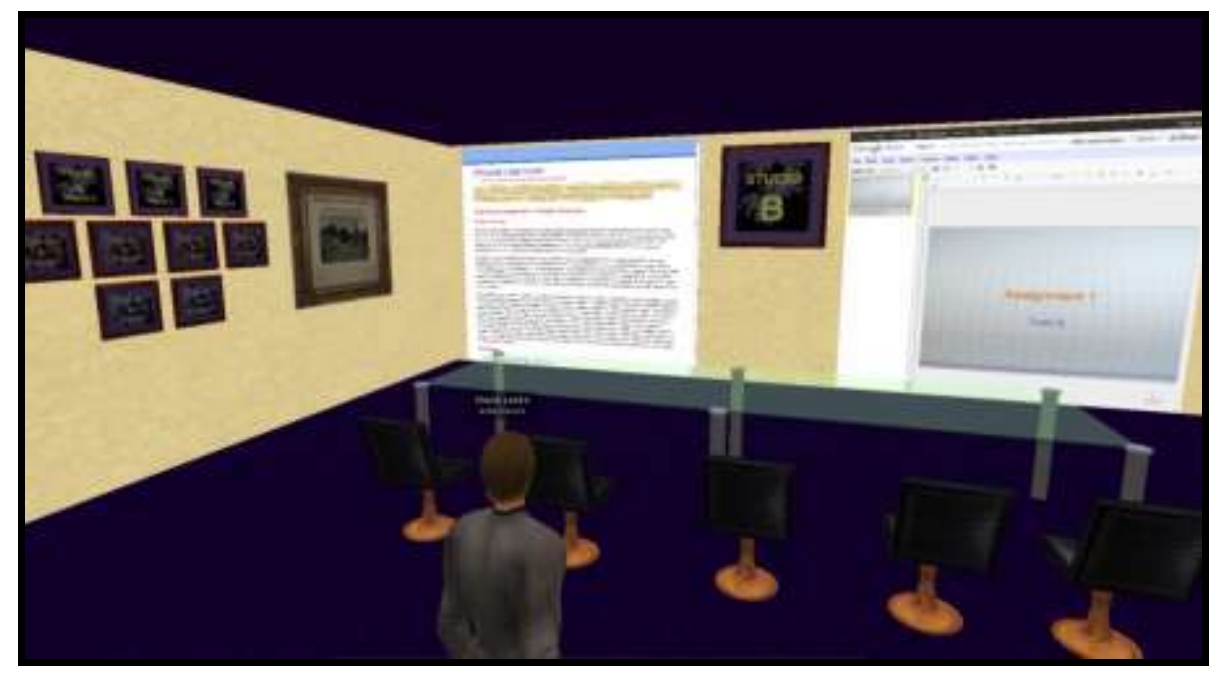

Figure 3: Virtual Team Studios (Sky Labs)

\section{Case Study Assignments and Role Play}

A significant portion of the student's course activity centered on applying the project management concepts presented in readings and lecture while utilizing an ongoing case study. For the case study there were (10) weekly project assignments throughout the course. Each student was assigned a role in the case study at the beginning of the course. Students were each given guidance on what was expected out of their role player and each role had (2) weekly sessions where they played that role. The role guidance came in the form of a 'Role Player Note 
Card' (see Figure 4) that was given to each role player. There were usually 3-4 role player's active during any given class session and 15-20 minutes of the class session was afforded to role play following team presentations.

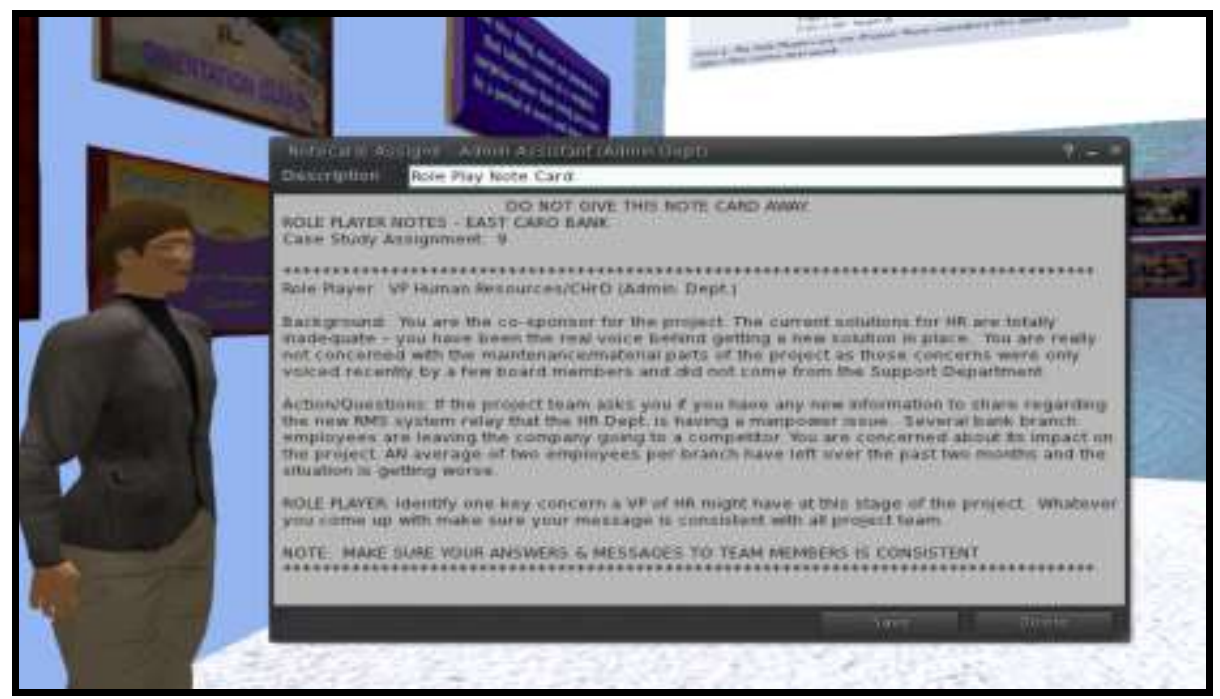

Figure 4: Case Study Role Card

Role play for the case study began week 3 of the course. The previous table (see Table 1) indicates which roles were assigned to a specific course week.

Within the case study there are several departments with multiple roles. Each project team is assigned to a department so that there is a never more than one team member assigned to role play on during a given class session. Additionally, project case study role play working spaces were developed specifically for student interaction based on the project case study (see Figure 5). During team collaboration time, role players would be available in their virtual office spaces to that project teams could meet with them and query them for further case content.

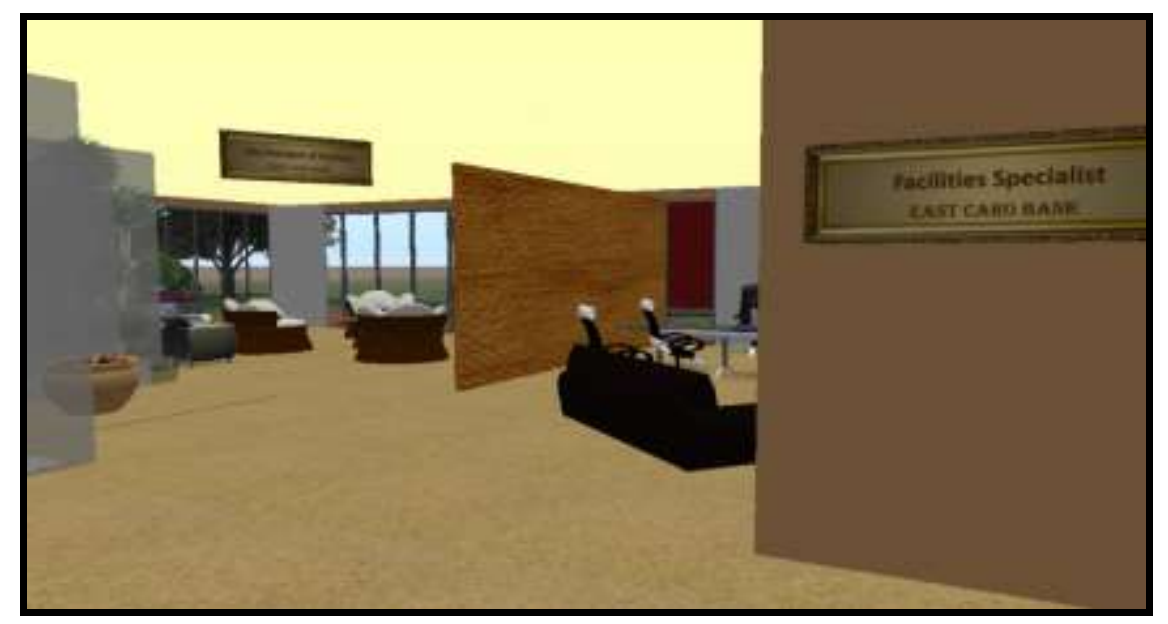

Figure 5: Case Study Role Play Workspaces 


\section{Preliminary Survey Results}

As indicated earlier, two survey assessment tools had been utilized. The scope of this article limits the discussion of the total survey results but a portion of the current results bares consideration here. The survey collection covered three-semester course offerings between 2011 and 2012. In each of these courses, the delivery involved not only lecture style learning and knowledge transfer, but also case study presentations, and virtual teaming, role play, and collaboration projects completed by the students.

The initial intent of the survey was to collect student perception data following completion of their first course experience within the virtual workspace. This same survey instrument had been used and pretested in a previous case study involving a smaller group of online graduate students. Over the course of the study period, the survey population consisted of (46) graduate students that were registered for the 3 different course offerings with (44) students actually completing the survey -- a return rate of $95.6 \%$. Preliminary results from the initial survey data were divided into four areas: 'Population Background', 'Learning Curve', 'Avatar Interaction', and 'Effectiveness of the Medium'.

Population Background. The survey results indicated that all but three of the respondents (93.2\%) had previously taken an online course for academic credit and that of those all but one respondent (97.7\%) had used some sort of online collaboration tool with their online course. These same results indicated that students had an increasing level of experience using virtual technology in their workplace since the start of the study. Interestingly, the study indicated that a growing number of students have had some experience in virtual world environments; more than a third $(34.1 \%)$ of graduate students stated they had operated in virtual worlds in the past.

Learning Curve. Preliminary survey results indicated that there was a minimal amount of time needed to adapt and learn to use the basic functions in Second Life. Only three of the survey respondents $(6.8 \%)$ reported that they took more than 2 hours to practice in the virtual world prior to the first class meeting. To assess collaborative challenges, students were asked if communicating in the virtual world (including local text chat and voice chat) was a difficult skill to master; to this query only six $(13.6 \%)$ of the respondents felt that communicating in the environment was difficult.

Avatar Interaction. Preliminary results indicated that the majority $(90.9 \%)$ of the students found the need for maintaining a Code of Conduct for virtual classroom behavior to be "important" for holding academic sessions in virtual environments. Additionally, most of the population $(90.9 \%)$ indicated they did not feel that the avatar's appearance was distracting in the work environment in any way. Another interesting response was that nearly half (45.4\%) of students indicated that the avatars did not have to closely resemble their human counterpart with (34.1\%) indicating they were undecided at this early juncture in their virtual world experience.

Perceived Effectiveness of the Medium. Preliminary results from the final area of the initial survey indicated that $(31.8 \%)$ of students reported that use of the virtual environment makes them more motivated to conduct online collaboration. 


\section{Closing Remarks and Moving Forward Considerations}

The virtual immersive environments today have the ability to simulate a dynamic and persistent three-dimensional interactive space that includes rich graphical life-like spaces that provide the end-user with a 'sense of presence' or 'being-there'. The collective research over the course of this study has concentrated on evaluating the use of virtually immersive environments not only as a learning media but also as a collaborative media for virtual project team interaction.

The purpose of this article was to present a role-play based approach to virtual project teaming and assessment that provides graduate students with collaborative working spaces, a context within which students can interact with their peers, and a structured approach for facilitators to evaluate the efforts of these virtual teams. Content and analytics for this study stemmed from data collected over the course of three semesters while offering a graduate-level Technology Project Management course. To offer this online course several virtual working spaces were utilized to present course lectures and discussions, and provide for virtual in-world team skills training.

Discussion included the incorporation of virtual role-play centered on the use of a contextually appropriate case study that spanned a period 10 of the 14 weeks of the semester-long course. The article also addressed both the structure of the course delivery, the case study and role play, and challenges faced in maximizing the immersive capabilities of these types of virtual environments all while ensuring that content delivery and required course assessments were being realized. The preliminary survey data provides us with a ground level idea of what questions should be asked next to determine the reasons for the responses here. Several points regarding these preliminary results are worth noting:

- It is felt that the previous experience in virtual worlds of over a third (34.1\%) of the students may have influenced other students in the class (with no previous virtual world experience) by providing support and advice informally to those new to the virtual environment. As a consequence this may have improved interpersonal communication trust among classmates and team members and should be more closely studied in the future. Trust is a critical virtual team characteristic and an area warranting further study. ${ }^{25,26}$

- With reference to the 'Learning Curve' category, the lesson taken from these early results indicates that providing a simple automated method for logging in and creating and operating an avatar, along with providing appropriate and clear training about the steps to do tends to ease the student's upfront learning curve. Additionally, it is perceived that easing the effort to learn a new technology like virtual world collaboration can result in a more systemic trust of the technology since there is not an overwhelming time commitment to learning how to operate in this new environment. However, the population is predominately made up of technology leaders so it could be presumed that they are more adapt at learning a new technological toolset. Broadening the population should aid in minimizing this as a plausible factor.

- With regard to the category of 'Avatar Interaction', results indicate there is a clear preference for parameters designed by the facilitator to give guidance to the activity and interaction between people in the environment. So, one advantage of establishing a basic 
Code of Conduct including avatar appearance appears to be that it avoids distraction based on appearance which should support trust building in the environment itself.

- A question remains, concerning the need for avatars to closely resemble their human proxies. This response needs further defining and consideration as it is not clear if students mean that avatars should still look human but do not need to look like the human for whom they stand as a proxy for or whether they in fact mean it is less important that an avatar even resemble a human being at all.

- Over the course of the study to date, it has been noted by several faculty observers that the implementation of role play appears to afford students more opportunity to interact closely with their peers giving them opportunities to visualize the project from a stakeholder's perspective. Research supports this notion ${ }^{20,19}$ and it is worth a more detailed observation and analysis as the study continues.

Further review of survey data conducted over the period of study to date, along with observational analysis, is the foundation for an ongoing discussion amongst practitioners who utilize this medium as an academic collaborative platform. Much of this data is just now beginning to provide us with information about student beliefs related to the development of trust and the quality of their team experience in the tested environment. Based on this current data collected to date, we are now in the process of reformulating more specific survey instruments and processes to gather objectively not just student reaction and beliefs regarding trust building and collaboration in the environment but to also ascertain whether actual trust building is exposed in the student's working environment. Additionally, further analysis regarding the case study and role play approaches is also warranted.

\section{References}

1. Is More Technology Better for Communication in International Virtual Teams? Duranti, Cleber Marchetti and de Almeida, Fernando Carvalho. 2012, International Journal of e-Collaboration, Volume 8, Issue 3, pp. 3652.

2. E-Collaboration and E-Commerce In Virtual Worlds: The Potential of Second Life and World of Warcraft. Kock, Ned. 2008, International Journal of e-Collaboration, Volume 4, Issue 3, pp. 1-13.

3. Dorr, Meena and Kelly, Kip. Developing Real Skills for Virtual Teams. Chapel-Hill, NC : UNC Executive Development 2011, 2011.

4. Five challenges to virtual team success: Lessons from Sabre, Inc. Kirkman, Bradley, et al., et al. 2002, Academy of Management Executive, Volume 16, Issue 3, pp. 67-79.

5. Something(s) old and something(s) new: Modeling drivers of global virtual team effectiveness. Maynard, M. Travis, et al., et al. 2012, Journal of Organizational Behavior, Volume 33, Issue 3, pp. 342-365.

6. A potential growth of education-industry collaboration. Ale Ebrahim, Nader, et al., et al. Kuala Lumpur, Malaysia : ICEED 2010, 2010. 2010 2nd International Congress on Engineering Education. pp. 7-9. 
7. Deloitte Development . Working in a virtual world Establishing highly effective virtual teams on information technology projects. Deloitte.com. [Online] December 19, 2011. http://www.deloitte.com/assets/DcomUnitedStates/Local\%20Assets/Documents/us_consulting_WorkingwithaVirtualWorld_080911.pdf.

8. Immersive Defined at Dictionary.com. reference.com. [Online] December 19, 2011. http://dictionary.reference.com/browse/immersive.

9. Miles, Mark W. US 7,868,847 B2 San Francisco, CA, 2011.

10. Stepping into the Internet: New Ventures in Virtual Worlds. Wasko, Molly, et al., et al. 2011, MIS Quarterly, Volume 35, Number 3, pp. 645-652.

11. Sud, Shivani and Pickering, Cynthia. COmputational Mobility and Virtual Worlds - Not Just Where You Work, But How You Work. [book auth.] Anand Prasad, John Buford and K. Vijay Gurbani. Future Internet Services and Service Architechtures. Denmark : River Publishers, 2011, pp. 207-226.

12. Preparing Teachers for the "Schools that Technology Built": Evaluation of a Program to Train Teachers for Virtual Schooling. Davis, N. and Roblyer, M. 2005, Journal of Research on Technology in Education, Volume 37, Number 4, pp. 399-409.

13. Future Directions of the Conferencing and Collaboration Field. Keary, Alfie and Redfern, Sam. 2012, International Journal of e-Collaboration, VOlume 8, Issue 2, pp. 1-24.

14. Creative expression in virtual worlds: Imitation, imagination, and individualized collaboration. . Ward, Thomas B. and Sonneborn, Marcene S. 2011, Psychology of Popular Media Culture, Volume 1(S), pp. 32-47.

15. Digital Mastery: The Skills Needed for Effective Virtual Leadership. Roy, Shelly R. 2012, International Journal of e-Collaboration, Volume 8, Issue 3, pp. 56-66.

16. Sense of Virtual Community: A Conceptual Framework and Empirical Validation . Koh, Joon and Kim, Young-Gul. 2004, International Journal of Electronic Commerce, Volume 8, Number 2, pp. 75-94.

17. A Framework for Adopting Collaboration 2.0 Tools for Virtual Group Decision Making . Turban, Efraim, Liang, Ting-Peng and Wu, Shelley P.J. 2011, Group Decision and Negotiation, Volume 2, Number 2, pp. 137-154.

18. E-Research Collaboration in Academia and Industry. Arinze, Bay. 2012, International Journal of eCollaboration, Volume 8, Issue 2, pp. 1-13.

19. Factors affecting students' decision to choose a traditional or role-play simulation based course format . Cox, Barbara, et al., et al. Louisville, Kentucky : International Conference on Computer Games 2012, 2012. Computer Games (CGAMES), 2012 17th International Conference. pp. 46-50.

20. Virtual Team Role Play Using Second Life for Teaching Business Process Concepts . Rudra, A, et al., et al. Koloa, Kauai, HI : Hawaii International Conference on System Sciences 2011, 2011. System Sciences (HICSS), 2011 44th Hawaii International . pp. 1-8.

21. Bryman, Alan. Social Research Methods (4th Edition). New York, NY : Oxford University Press, 2012.

22. Babbie, Earl. Survey Research Methods. Belmont, CA : Wadsworth Publishing Company, 1990.

23. Heagney, Joseph. Fundamentals of Project Management, 4th Edition. New York, NY : AMACOM, 2012.

24. Meredith, Jack R. and Mantel, Jr., Samuel j. Project Management: A Managerial Approach. Hoboken, NJ : John Wiley and Sons, Inc., 2012. 
25. The Impact of Training on Virtual Project Teams: A TIP Investigation. Beranek, Peggy and Clairborne, M. Cathy. 2012, International Journal of Information Technology Project Management, Volume 3, Issue 1, pp. 113.

26. The Role of Communication and Trust in Global Virtual Teams: A Social Network Perspective. Sarker, Saonee, Ahuja, Manju and Kirkeby, Sarah. 2011, Journal of Management Information Systems , pp. 273-310.

27. Czaja, Ronald and Blair, Johnny. Designing Surveys: A Guide to Decisions and Procedures. Thousand Oaks, CA : Pine Forge Press, 1995.

28. Korolov, Maria. Virtual world usage accelerates. Hypergrid Business. July 28, 2011. 